\title{
Qualidades psicométricas no papel da Escala de Habilidades de Vida Independente de pacientes psiquiátricos (ILSS-BR)
}

\author{
Marina Bandeira ${ }^{1}$ \\ Lúcia Abelha Lima² \\ Sylvia Gonçalves ${ }^{3}$
}

Recebido: 27/2/2003 Aceito: 23/7/2003

\begin{abstract}
RESUMO
Esta pesquisa teve como objetivo fazer a análise psicométrica de fidedignidade do teste e do reteste da versão brasileira da escala Independent Living Skills Survey (ILSS-BR), que avalia a autonomia de pacientes crônicos em diversas áreas do funcionamento social. Um estudo prévio havia mostrado que esta escala apresentava qualidades psicométricas adequadas de consistência interna das suas subescalas, assim como validade discriminante e validade de construto, sem ter avaliado, no entanto, sua estabilidade temporal. Os resultados da presente pesquisa mostraram que a escala ILSS-BR apresentou coeficientes de correlação significativos entre os escores do teste e do reteste, para todas as suas subescalas e para o escore global. Esses resultados indicam que o ILSS-BR é um instrumento de medida que apresenta estabilidade temporal, mostrando-se, portanto, um instrumento fidedigno para ser utilizado no planejamento e na avaliação de programas relacionados à reabilitação psicossocial de pacientes psiquiátricos, no contexto brasileiro.

Unitermos: Escala de medida; Qualidades psicométricas; Habilidades de vida cotidiana; Pacientes psiquiátricos; Autonomia

ABSTRACT

Independent Living Skills Survey of Psychiatric Patients (ILSS-BR)

This research analyzed the psychometric property of test-retest reliability of the Brazilian version of the Independent Living Skills Survey scale (ILSS-BR), developed to provide global assessments of functional living skills of chronically mentally ill individuals. Previous research have demonstrated that the ILSS-BR had good psychometric properties of reliability and validity, in terms of the internal consistency of its sub-scales, construct validity of the scale and discriminant validity, but have not analyzed its temporal stability. The results of the present research showed significant correlation coefficients between the test and the retest scores of the ILSS-BR, for each of its sub-scales and for the global score. This result demonstrated that the ILSS-BR has temporal stability and is a reliable scale to be used in the planning and evaluation of psychosocial rehabilitation program for psychiatric patients, in brazilian context.
\end{abstract} Keywords: Scale; Psychometric properties; Independent living skills; Psychiatric patients.

Psicóloga, Pesquisadora e Docente da Universidade Federal de São João del Rei (UFSJ), Doutora pela Université de Montréal e PósDoutora pelo Psychosocial Research Centre da McGill University e pelo Centre de Recherche Fernand Séguin, Canadá.

2 Psiquiatra e Pesquisadora do Núcleo de Pesquisa do Instituto de Assistência à Saúde Juliano Moreira e Doutora em Saúde Pública da Escola Nacional de Saúde Pública/Fundação Oswasdo Cruz.

3 Psicóloga e Pesquisadora do Núcleo de Pesquisa do IMASJM e Especialista em Saúde Mental da Escola Nacional de Saúde Pública/ Fundação Oswaldo Cruz.

Endereço para correspondência:

Laboratório de Pesquisa em Saúde Mental (LAPSAM) - Departamento de Psicologia da UFSJ

Praça Dom Helvécio, 74 - São João del Rei CEP 36301-160

E-mail: bandeira@ufsj.edu.br

Site: www.ufsj.edu.br/saudemental 


\section{Introdução}

A desinstitucionalização psiquiátrica provocou uma mudança no conceito de tratamento dos pacientes psiquiátricos, de forma a ampliar o enfoque anteriormente limitado a intervenções terapêuticas de redução da sintomatologia, para uma abordagem mais ampla que coloca em destaque na promoção da qualidade de vida dos pacientes na comunidade. Três abordagens principais se destacaram, segundo Mercier (1994), na conceituação da qualidade de vida, relacionando-se a três dimensões distintas: 1 . as condições objetivas de vida e o grau de satisfação subjetiva dos pacientes em relação a elas; 2. o bem-estar psicológico dos pacientes (afeto positivo e negativo, auto-estima, etc.) e 3 . o repertório de habilidades e competências, capacidades e limites funcionais, tais como as habilidades motoras/instrumentais, intelectuais, sociais e sensoriais dos pacientes na vida cotidiana.

Estas dimensões estão inter-relacionadas, pois as habilidades de vida cotidiana possibilitam o desenvolvimento do sentimento de competência, domínio ou autonomia nas atividades do dia-a-dia, que contribuem para a qualidade de vida subjetiva das pessoas (Mercier, 1994). Um dos objetivos de diversos tipos de programas de reabilitação psicossocial e acompanhamento intensivo dos pacientes na comunidade é justamente $o$ de desenvolver as habilidades que resultam em maior grau de autonomia, em diversas áreas da vida cotidiana, tais como atividades ocupacionais, lazer, saúde, nutrição, finanças, autocuidados, uso de transporte, atividades domésticas, etc. (Liberman et al., 1993; Wallace e Liberman, 1985; Bandeira et al., 1998). Revisões de literatura nesta área mostraram que os treinamentos de habilidades sociais e da vida cotidiana são procedimentos eficazes de reabilitação psiquiátrica (Benton e Schroeder, 1990; Corrigan, 1991; Donahoe e Driesienga, 1988).

Para isso, entretanto, é importante avaliar, por meio de instrumentos de medida válidos e fidedignos, o nível de habilidades dos pacientes, nas diversas áreas da vida cotidiana, a fim de identificar objetivos mais pertinentes para os programas de reabilitação e monitorar posteriormente os efeitos desses programas na vida dos pacientes.

Um dos instrumentos de medida que visam avaliar o grau de habilidades da vida cotidiana de pacientes psiquiátricos é o Independent Living Skills Survey - ILSS, elaborado por Wallace et al. (1985) e descrito por Wallace (1986). Essa escala de avaliação foi submetida a uma adaptação transcultural para o Brasil, seguindo os procedimentos metodológicos recomendados pela Organização Mundial de Saúde
(OMS, 1996) e por Vallerand (1989), e a versão brasileira resultante foi submetida a uma validação de suas propriedades psicométricas (Lima et al., 2002), recebendo a denominação de ILSS-BR. O estudo de validação evidenciou qualidades psicométricas adequadas, de validade e fidedignidade, para a versão brasileira da escala. O estudo da fidedignidade, em particular, avaliou a consistência interna dos itens das subescalas do ILSS-BR, demonstrando que os itens apresentavam uma boa homogeneidade ou consistência interna nas suas subescalas, tendo obtido valores elevados do coeficiente alfa de Cronbach (entre 0,67 a 0,96 ), considerados adequados para o número de itens das subescalas (Gulliksen, 1950; Martinez, 1995).

Não foi realizada, entretanto, no estudo de validação do ILSS-BR, a avaliação de sua estabilidade temporal ou fidedignidade do teste e do reteste. O estudo da fidedignidade de uma escala envolve não somente sua consistência interna, mas também sua estabilidade temporal. Para preencher essa lacuna, a presente pesquisa tem como objetivo fazer o estudo da estabilidade temporal do ILSS-BR, por meio do procedimento teste e reteste.

\section{Método}

\section{Local de estudo}

O Instituto Municipal de Assistência à Saúde Juliano Moreira (IMASJM) é um hospital psiquiátrico localizado em Jacarepaguá, RJ, fundado em 1924, que tem como clientela 905 pacientes institucionalizados por muitos anos e que perderam seus vínculos familiares e sociais em decorrência dos longos anos de institucionalização. O IMASJM tem a característica de um pequeno bairro, pois atualmente residem no seu perímetro cerca de 20 mil moradores, fruto de invasões das terras da antiga Colônia Juliano Moreira (Gonçalves et al., 2001).

O instituto é composto de seis unidades assistenciais: $173(19 \%)$ pacientes no Núcleo Franco da Rocha (NFR); 169 (19\%) no Núcleo Ulisses Viana (NUV); 344 (38\%) no Núcleo Teixeira Brandão; 102 (11\%) no Núcleo Rodrigues Caldas (NRC) e 64 (7\%) no Pavilhão Agrícola (PA) e Centro de Reabilitação e Integração Social (CRIS) com $53(6 \%)$ pacientes. As diversas unidades do IMASJM oferecem atividades em oficinas, atendimento terapêutico, acompanhamento no Programa de Recursos Individuais, Residências Terapêuticas e Lares de Acolhimento e atividades de lazer. 


\section{Participantes}

Participaram desta pesquisa uma amostra de 49 sujeitos selecionados aleatoriamente da população de pacientes da instituição. Os participantes são pacientes de duas unidades do IMASJM, o Núcleo Franco da Rocha (24 pacientes do sexo feminino) e o Núcleo Ulisses Viana (25 pacientes do sexo masculino). Tratase de uma subamostra da população de pacientes que participaram do estudo original de validação da escala ILSS-BR (Lima et al., 2002).

A subamostra da presente pesquisa é constituída de 25 homens $(51,02 \%)$ e 24 mulheres $(48,98 \%)$, de faixa etária entre 38 e 86 anos, com uma média de idade de 63,31 anos $(\mathrm{sd}=11,09)$. O tempo médio de internação desses pacientes é de 36,31 anos $(\mathrm{sd}=12,32)$. $\mathrm{O}$ nível de escolaridade é baixo, com $34,7 \%$ de pacientes analfabetos e $28,6 \%$ apenas alfabetizados. Quanto ao perfil diagnóstico, $57,1 \%$ dos participantes têm diagnóstico de esquizofrenia, transtornos esquizotípicos ou transtornos delirantes; $18,3 \%$ têm retardo mental; $10,2 \%$ têm epilepsia e $14,4 \%$ têm outros diagnósticos.

\section{Instrumento de medida}

A versão original da escala, Independent Living Skills Survey (ILSS), foi elaborada por Wallace et al. (1985) e relatada por Wallace (1986). A escala original contém 112 itens, distribuídos em 9 subescalas, que avaliam as habilidades de vida independente de pacientes psiquiátricos com distúrbios severos e persistentes. Possui duas versões, uma delas construída para ser aplicada diretamente aos próprios pacientes e a outra para ser aplicada a um informante, seja ele um membro da equipe de saúde mental envolvido no atendimento ao paciente ou um membro da família do paciente. A escala original na língua inglesa apresenta propriedades psicométricas adequadas de consistência interna, com coeficientes alfa de Cronbach variando de 0,67 a 0,84 . A avaliação da fidedignidade pelo método da correlação entre as duas metades das subescalas apresentou coeficientes de correlação entre 0,63 e 0,89. Apresenta igualmente índices adequados de validade concomitante, tendo sido correlacionada positivamente com as subescalas de funcionamento social da escala NOSIE (Honifeld et al., 1966), a qual avalia o funcionamento e a sintomatologia de pacientes psiquiátricos (Wallace, 1986).

A versão da escala adaptada e validada para o Brasil (Lima et al., 2002) foi aquela a ser aplicada por um informante. O estudo de tradução e adaptação da escala foi realizado pelo Núcleo de Pesquisa do IMASJM e a coleta de dados foi feita nas unidades assistenciais da instituição. A análise das qualidades psicométricas das escalas foi realizada pelo Laboratório de Pesquisa em Saúde Mental da Universidade Federal de São João del Rei (UFSJ), MG. O Inventário de Habilidades de Vida Independente foi submetido a uma tradução e a uma retrotraduação (backtranslation), assim como a um estudo piloto sendo avaliado por uma comissão de especialistas que tem por finalidade adaptá-lo ao contexto brasileiro.

A versão brasileira da escala, denominada ILSSBR, possui 84 itens distribuídos em 9 subescalas que avaliam o funcionamento dos pacientes psiquiátricos em nove áreas da vida cotidiana, em termos da freqüência em que eles apresentam as habilidades básicas para funcionar de forma independente na comunidade. As nove áreas avaliadas pela escala referem-se a atividades relacionadas a: alimentação, cuidados pessoais, atividades domésticas, preparo e armazenamento dos alimentos, saúde, lazer, emprego e transporte. A ILSS-BR avalia, em uma escala do tipo Likert de 5 pontos (nunca, algumas vezes, com freqüência, na maioria das vezes, sempre), a freqüência com que o paciente realizou, no último mês, as atividades cotidianas necessárias ao seu funcionamento independente na comunidade.

A ILSS-BR mostrou-se uma escala com qualidades psicométricas de validade e fidedignidade satisfatórias, no que se refere à consistência interna das suas subescalas, assim como à sua validade discriminante e validade de construto (Lima et al., 2002).

\section{Procedimento}

A reaplicação da escala ILSS-BR na subamostra selecionada foi iniciada após um intervalo de duas semanas depois do término da primeira aplicação da escala, efetuada no contexto do estudo inicial de sua validação. Esta reaplicação recebeu aprovação da comissão de ética em pesquisa do IMASJM, garantindose o consentimento, o sigilo e o anonimato das respostas.

O reteste foi realizado no período de duas semanas, por meio de entrevistas estruturadas realizadas pelos mesmos entrevistadores envolvidos no primeiro estudo. As entrevistas foram feitas com os funcionários da unidade que mais conheciam os pacientes nas suas atividades diárias. Os entrevistadores foram uma psiquiatra e uma psicóloga, co-autoras desse estudo. O treinamento dos entrevistadores foi constituído de três módulos e teve a duração de 12 horas: 1 . discussão da escala, 2. aplicação piloto da escala e 3. painéis de discussão.

Durante as entrevistas, o entrevistador lia inicialmente as instruções sobre as alternativas possíveis de respostas e, em seguida, lia cada um dos itens da escala. As respostas de cada respondente eram anotadas pelo entrevistador no próprio questionário. 


\section{Resultados}

A tabela 1 apresenta as médias e desvios padrão dos escores obtidos no teste e no reteste, assim como os coeficientes de correlação de Pearson para cada subescala e para o escore global da ILSS-BR.

Pode-se observar, na tabela 1, que todas as subescalas do ILSS-BR apresentaram correlações significativas entre os escores obtidos na primeira e na segunda aplicações (teste-reteste). Os coeficientes de correlação mais elevados foram obtidos para as subescalas de atividades domésticas, preparo e manutenção dos alimentos, administração do dinheiro e cuidados pessoais. Em seguida, observam-se os escores das subescalas que se referem ao uso dos meios de Transporte, às atividades relacionadas à Saúde, às atividades de Lazer, aos hábitos alimentares e às atividades referentes à busca ou manutenção de Emprego. O escore global da escala ILSS-BR apresentou igualmente uma correlação significativa elevada entre os escores do teste e do reteste.

\section{Discussão}

Os resultados obtidos na presente pesquisa mostraram que a versão brasileira do Inventário de Habilidades de Vida Independente, ILSS-BR, apresentou índices adequados de fidedignidade, em termos de estabilidade temporal, avaliados por meio do procedimento teste-reteste. Os valores obtidos foram próximos aos encontrados por Wallace na versão original da ILSS em inglês, que variaram de 0,63 a 0,89 (Wallace, 1986). Os coeficientes de correlação obtidos aqui para os escores do teste e do reteste das subescalas variaram entre 0,51 e 0,91 , o que constitui uma faixa de valores ligeiramente superiores aos valores encontrados para a versão de língua francesa-canadense, que variaram entre 0,48 e 0,85 (Cyr et al., 1992). Os valores obtidos na presente pesquisa foram também superiores aos encontrados na versão mais recente de língua inglesa, que variaram entre 0,43 e 0,91 (Wallace et al., 2000).

$\mathrm{O}$ valor mais baixo do coeficiente de correlação teste-reteste obtido na presente pesquisa se referiu à subescala de atividades de procura e manutenção de emprego, o que concorda com os resultados obtidos para a versão de língua inglesa mais recente (Wallace et al., 2000). Esses dados não podem ser comparados com o estudo da versão francesa-canadense, uma vez que não foram calculados os coeficientes de correlação teste-reteste para as escalas de procura e manutenção de emprego, em virtude do número reduzido de sujeitos. Neste último estudo, foi a subescala de uso dos meios de transporte que apresentou o valor mais baixo de correlação teste-reteste.

Os resultados obtidos na presente pesquisa servem para ampliar o conhecimento a respeito das qualidades psicométricas da escala ILSS-BR, cujos dados iniciais já haviam constatado a consistência interna dos itens de cada subescala, sem no entanto ter avaliado sua estabilidade temporal. Estes dois resultados, tomados em conjunto, confirmam que o ILSS-BR possui qualidades psicométricas adequadas de fidedignidade, tanto em termos de consistência interna quanto de estabilidade temporal.

A escala ILSS-BR constitui, portanto, um instrumento fidedigno para ser utilizado no planejamento e na avaliação de programas relacionados à reabilitação psicossocial de pacientes psiquiátricos no contexto brasileiro.

Tabela 1 Médias e desvios padrão do teste e do reteste e coeficientes de correlação de Pearson para as subescalas e escore global da ILSS-BR

\begin{tabular}{lccccc}
\hline Subescalas & \multicolumn{2}{c}{ Médias } & \multicolumn{2}{c}{ Desvios padrão } \\
& Teste & Reteste & Teste & Reteste & Correlação \\
\hline Atividades domésticas & 1,78 & 1,82 & 1,53 & 1,54 & $0,91^{* *}$ \\
Preparo e manutenção & 1,66 & 1,72 & 1,36 & 1,31 & $0,89^{* *}$ \\
de alimentos & & & & \\
Administração do dinheiro & 1,95 & 1,92 & 1,20 & 1,31 & $0,88^{* *}$ \\
Cuidados pessoais & 2,09 & 2,08 & 1,04 & 0,96 & $0,86^{* *}$ \\
Uso dos meios de transporte & 1,38 & 1,15 & 1,13 & 1,18 & $0,79^{* *}$ \\
Atividades referentes à saúde & 2,35 & 2,42 & 0,96 & 1,00 & $0,77^{* *}$ \\
Atividades de lazer & 0,99 & 1,14 & 0,72 & 0,76 & $0,75^{* *}$ \\
Hábitos alimentares & 2,96 & 3,01 & 0,87 & 0,90 & $0,71^{* *}$ \\
Atividades referentes a emprego & 0,23 & 0,46 & 0,40 & 0,79 & $0,51^{* *}$ \\
Escore global & 1,68 & 1,69 & 0,84 & 0,81 & $0,89^{* *}$ \\
\hline
\end{tabular}

$* *<0,001$ 


\section{Referências bibliográficas}

Bandeira, M.; Gelinas, D.; Lesage, A. - Desinstitucionalização: o programa de acompanhamento intensivo na comunidade. Jornal Brasileiro de Psiquiatria 12: 627-40, 1998.

Benton, M.K.; Schroeder, H.E. - Social skills training with schizophrenics: a meta-analytic evaluation. $J$ Cons and $\mathrm{Cl}$ Psychology 58: 741-7, 1990.

Corrigan, P.W. - Social skills training In adult psychiatric populations: a meta-analysis. J. of Beh. Ther. and Exp. Psychology 22: 203-10, 1991

Cyr M.; Lesage, A.D.; Toupin, J. - L'évaluation des problèmes psychosociaux chez des personnes ayant des problèmes psychiatriques graves. Documento Inédito, Relatório de Pesquisa. ISBN 2-921486-00-8, 1992.

Donahoe, C.P.; Driesienga, S.A. - A review of social skills training with chronic mental patients, In: Hersen, M.; Eisler, R.M.; Miller, P.M. (eds.): Progress in Behavioral Modification. Sage, Newbury Park, CA, pp.131-164, 1988.

Gonçalves, S; Fagundes, P.; Lovisi, G.; Lima. L.A. - Avaliação das limitações no comportamento social de pacientes psiquiátricos de longa permanência. Ciência e Saúde Coletiva 6(1): 105 13,2001

Gulliksen, H. - Theory of mental test. John Wiley, New York, 1950.

Honifeld, G.E.; Roderic, D.; Klett, J.C. - Nosie-30: A treatment sensitive ward behavior scale. Psychologial Reports 19: 1802, 1966.

Liberman, R.P.; Wallace, C.J.; Blackwell, C.A.; ECKMAn, T.A.; Vaccaro, J.V.; KUEHNEL, T.G. - Innovations in skills training for the seriously mentally ill: The UCLA Social and Independent Living Skills modules. Innovations and Research 2: 43-60, 1993.

Lima, L.A.; Bandeira, M.; Gonçalves, S. - Validação Transcultural do Inventário de Habilidades de Vida Independente para Pacientes Psiquiátricos - (ILSS-BR). Jornal Brasileiro de Psiquiatria (no prelo).
Martinez, A.R - Psicometria: teoria de los tests psicológicos y educativos. Editorial Sintesis, Madrid, 1995.

Mercier, C. - La methodologie de l'évaluation de la qualité de vie subjective en psychiatrie. In: Terra, J.L. (ed.): Qualité de vie subjective et santé mentale: aspects conceptuels et méthodologiques. Ellipses, Paris, pp. 93-109, 1994.

Ruggeri, M. - Patients'and relatives'satisfaction with psychiatric services: the state of the art of its measurement. Soc Psychiatry Psychiatr Epidemiology 29: 212-27, 1994.

VALLERAND, R. - Vers une methodologie de validation trans-culturelle de questionnaires psychologiques. Canadian Psychology 30: 662-80, 1989.

Wallace, C.J. - Functional Assessment in Rehabilition. Schizophrenia Bulletin 12: 604-23, 1986.

Wallace, C.J.; Kochanowicz, N.; Wallace, J. - Independent Living Skills Survey. Unpublished manuscript, Mental Health Clinical Research Center for the Study of Schizophrenia. West Los Angeles VA Medical Center, Rehabilitation Medicine Service (Brentwood Division), Los Angeles, CA 90073, 1985 .

Wallace, C.J.; Boone, S.E.; Donahue, P.C.; Foy, D.W. - The cronically mentally disabled: Independent living skills training. In: Barlow, D.H. (ed.): Clinical Handbook of Psychological Disorders. Guilford, New York, pp. 462-501, 1985.

Wallace, C.J.; Liberman, R.P. - Social skills training for patients with schizophrenia: a controlled clinical trial. Psychiatry Research 15: 239-47, 1985

Wallace, C.J.; Liberman, R.P.; Tauber, R.; Wallace, J. - The Independent Living Skills Survey: A compreehensive measure of the community functioning of severely and persistently mentally ill individuals. Schizophrenia Bulletin 26 (3): 63258,2000

World Health Organization. Who-satis Consumer's and caregivers' satisfaction with Mental Health Services: a Multisite Study. Division of Mental Health, Geneva, 1996.

\section{O arquivo disponível sofreu correções conforme ERRATA publicada no Volume 30 Número 5 da revista.}

